\title{
The lithium effects on morphology and apoptosis in hepatocellular carcinoma cells
}

\author{
Iuliia Taskaeva \\ Laboratory of ultrastructural research, \\ Research Institute of Clinical and \\ Experimental Lymphology - Branch of \\ the Institute of Cytology and Genetics, \\ Siberian Branch of the Russian \\ Academy of Sciences; \\ Laboratory of boron-neutron capture \\ therapy, Department of Physics, \\ Novosibirsk State University \\ Novosibirsk, Russia \\ inabrite@yandex.ru \\ Viktoriia Makarova \\ Laboratory of ultrastructural research, \\ Research Institute of Clinical and \\ Experimental Lymphology - Branch of \\ the Institute of Cytology and Genetics, \\ Siberian Branch of the Russian \\ Academy of Sciences \\ Novosibirsk, Russia \\ shedina_vika@mail.ru
}

\author{
Izabella Gogaeva \\ Laboratory of ultrastructural research, \\ Research Institute of Clinical and \\ Experimental Lymphology - Branch of \\ the Institute of Cytology and Genetics, \\ Siberian Branch of the Russian \\ Academy of Sciences; \\ Department of Natural Sciences, \\ Novosibirsk State University \\ Novosibirsk, Russia \\ i.gogaeva@g.nsu.ru
}

\author{
Nataliya Bgatova \\ Laboratory of ultrastructural research, \\ Research Institute of Clinical and \\ Experimental Lymphology - Branch of \\ the Institute of Cytology and Genetics, \\ Siberian Branch of the Russian \\ Academy of Sciences \\ Novosibirsk, Russia \\ n_bgatova@ngs.ru
}

\author{
Natalia Obanina \\ Laboratory of ultrastructural research, \\ Research Institute of Clinical and \\ Experimental Lymphology - Branch of \\ the Institute of Cytology and Genetics, \\ Siberian Branch of the Russian \\ Academy of Sciences; \\ Section of cytology and genetics, \\ Department of Natural Sciences, \\ Novosibirsk State University \\ Novosibirsk, Russia \\ n.obanina@g.nsu.ru
}

\begin{abstract}
Hepatocellular carcinoma (HCC) is characterized dysregulation of cell death mechanisms, and the imbalance of pro- and anti-apoptotic signals. The development of HCC is accompanied by genetic mutations in the signaling pathways involved in the cell proliferation, growth and death. The molecular changes in apoptosis signaling in $\mathrm{HCC}$ determine the requirement for targeted chemotherapy to increasing apoptosis in $\mathrm{HCC}$ cells. The aim of this study was to assess the ability of lithium to influence on the hepatocellular carcinoma29 (HCC-29) cells apoptosis in vivo. Light and transmission electron microscopy, and immunofluorescence staining were used to evaluate of apoptosis development in HCC-29 cells after administration of $20 \mathrm{mM}$ lithium carbonate. It was revealed that lithium extremely increased the pro-apoptotic proteins Bad and caspase- 3 expression, and decreased the anti-apoptotic protein Bcl-2 expression. These results indicate that lithium carbonate induces apoptosis pathways in HCC-29 cells. Lithium administration can enhance pro-apoptotic chemotherapeutic drugs potential and overcome the resistance of tumor cells to apoptosis in HCC.
\end{abstract}

Keywords - hepatocellular carcinoma, lithium, morphology, apoptosis, electron microscopy

\section{Introduction}

Hepatocellular carcinoma (HCC) is a highly malignant type of liver cancer characterized by the presence of multiple genetic mutations in the Wnt/beta-catenin, p53, PI3K/Ras signaling pathways, as well as in the processes of oxidative and endoplasmic reticulum stress [1]. These pathways are the main intracellular signaling cascades involved in the proliferation, growth and death of tumor cells. Furthermore, HCC is characterized by molecular changes that affect on apoptosis processes: mutations in the P53 tumor suppressor gene (TP53), increased expression of transforming growth factor (TGF- $\beta$ ) and the anti-apoptotic phenotype, resistance to extrinsic apoptosis [2]. The dysregulation of cell death and proliferation mechanisms, as well as the imbalance of pro- and anti-apoptotic signals in HCC require the targeted chemotherapy to enhancing HCC apoptosis [3]. The effects of lithium on carcinogenesis and tumor progression are currently being actively studied [4]. Lithium implements its effect mainly by inhibiting glycogen synthase kinase $3 \beta$ (GSK-3 $\beta$ ), an enzyme that regulates the proliferation, differentiation and apoptosis of tumor cells [5]. We have previously shown that lithium salts decrease viability and stimulate apoptosis in hepatocellular carcinoma-29 cells in vitro $[6,7]$. The aim of this study was to investigate the lithium effects on cell morphology and HCC-29 apoptosis in vivo.

\section{Methods and algorithms}

HCC-29 was obtained by the researchers of the Institute of Cytology and Genetics, and was kindly provided for our study [8]. Mice were maintained at a constant room temperature (23 $\left.{ }^{\circ} \mathrm{C}\right)$ with a natural day/night light cycle in a conventional animal colony. Standard laboratory food and water were provided. Male CBA mice of 6-8 weeks of age, with weights of 18-20 g were used in experiment. For tumor induction, 1 . $10^{6}$ HCC-29 cells were transplanted into the abdominal cavity, after 10 days, ascitic fluid was removed and $2 \cdot 10^{6}$ HCC-29 cells suspended in $100 \mu \mathrm{L}$ of PBS were injected into the right thigh muscle. Mice were randomly divided into two experimental groups (five mice in each). Lithium $\left(\mathrm{Li}_{2} \mathrm{CO}_{3}\right)$ was delivered in $100 \mu \mathrm{L}$ volumes given intramuscularly along periphery of the tumor every day. Experimental groups were as follows: the mice with intact tumor (Control) and mice, received $20 \mathrm{mM}$ lithium carbonate (LC). All mice were euthanized by cervical dislocation under overdose of anesthesia on the 23rd day of the experiment. Animal testing was performed in accordance with Directive 2010/63/EU. Transmission electron microscopy (TEM). Tumor tissue was fixed with a $4 \%$ paraformaldehyde and then incubated with $1 \%$ osmium tetroxide $(\mathrm{OsO} 4)$ at $4{ }^{\circ} \mathrm{C}$ for $1 \mathrm{~h}$. The tumor tissue 
was then incubated with $1 \%$ uranyl acetate after which it were further processed at the JEM 1400 electron microscope (JEOL, Japan). Microscopic analysis was carried out at the Multiple-access Center for Microscopy of Biological Subjects (Institute of Cytology and Genetics, Novosibirsk, Russia). Immunofluorescent staining $(I F-F)$. Frozen sections of tumor tissue $(10 \mu \mathrm{m})$ were analyzed by immunofluorescence staining using anti-Bad (ab32445, Abcam, UK), anti-Bcl-2 (ab692, Abcam, UK) and anti-caspase-3 (ab13847, Abcam, UK) primary antibodies. Alexa Fluor 488-conjugated antirabbit IgG (ab150077, Abcam, UK) and Alexa Fluor 594conjugated anti-mouse IgG (ab150108, Abcam, UK) were used to detect the corresponding primary antibodies. Images were analyzed using an Axio Observer Z1 (Zeiss, Germany) fluorescence microscope at $\times 400$ final magnification. Fourteen fields per group were captured (total area was 0.08 $\mathrm{mm}^{2}$ for each group). The number of Bad-, Bcl-2- and caspase-3 positive cells was counted and analyzed using ImageJ software (National Institutes of Health, Bethesda, MD). Statistical analysis. Data are presented as mean (M) \pm standard deviation (SD). Mann-Whitney nonparametric tests were used to assess differences by statistical package Statistica 6.0 (StatSoft, USA). Statistically significant differences were considered at $\mathrm{P}<0.05$.

\section{Results}

An ultrastructural study of tumor tissue revealed apoptotic changes in HCC-29 cells after administration of $20 \mathrm{mM}$ lithium carbonate. In cancer cells fragmentation of the nucleus and condensation of chromatin, a decrease in cell volume and cytopodia, as well as plasma membrane bleeding were detected. These changes are typical morphological manifestations of apoptosis. After immunofluorescence staining of tumor tissue cryosections, it was found that the introduction of lithium significantly increased the number of Bad-positive and caspase-3-positive cells (Table 1). In addition, the number of Bcl-2 positive cells decreased significantly after lithium administration. On hematoxylinstained paraffin sections, necrosis zones were calculated for each group. There were no significant differences between the groups: in the control group, the percentage of such zones was $5.41 \pm 5.29$, and after lithium administration $-5.44 \pm 3.3 \%$. Apoptosis is a process of regulated cell death that develops as a result of intracellular changes (intrinsic pathway of apoptosis) or changes in the microenvironment of the cell (extrinsic pathway of apoptosis) [9]. The intrinsic pathway of apoptosis is initiated by disturbance of intracellular homeostasis, leading to permeabilization of the outer mitochondrial membrane, which occurs under the control of the regulating apoptosis Bcl-2 proteins family [10]. The extrinsic apoptosis pathway is triggered mainly by death receptors, which results in the formation of a death-inducing signaling complex (DISC) and activation of caspase-8, -10, 3 and others [10]. The results obtained in this study demonstrate the possible ability of lithium to influence on the mitochondrial membranes permeability and participate in the intrinsic and probably extrinsic apoptotic pathways.

On the various HCC cell lines, lithium has been shown to induce apoptosis [11]. An increase in caspase-3, -8, and p53 levels and TRAIL-induced apoptosis in HCC cells has also been identified [12]. Thus, the results obtained are consistent with the literature data on the lithium effects on HCC cells apoptosis.
TABLE I.

\begin{tabular}{|l|c|c|c|}
\hline \multirow{2}{*}{ Group } & \multicolumn{3}{|c|}{ Apoptotic Markers } \\
\cline { 2 - 4 } & Bad & Caspase-3 & Bcl-2 \\
\hline Control & $29.57 \pm 10.44$ & $4.71 \pm 3.41$ & $38.29 \pm 21.42 * *$ \\
\hline $\begin{array}{l}\text { Lithium } \\
\text { carbonate }\end{array}$ & $60.93 \pm 45.9 * *$ & $21.57 \pm 17.89 *$ & $16.79 \pm 8.65$ \\
\hline \multicolumn{4}{|c}{} \\
$* \mathrm{P}<0.05 ; * * \mathrm{P}<0.005$
\end{tabular}

\section{Conclusion}

GSK-3 $\beta$ is the main intracellular target of lithium involved in a multiple signaling pathways associated with tumor cell growth and proliferation, cell death and survival (PI3K/Akt/mTORC1, Ras/Raf/MEK/ERK, Wnt/beta-catenin, Hedgehog and others). Most likely, lithium affects the development of apoptosis due to inhibition of GSK-3 $\beta$, nevertheless, further studies of the lithium-related apoptotic changes in HCC cells are required. The molecular features of HCC that contribute to the apoptosis resistance determine the relevance of further research and the development of new strategies for enhancing apoptosis in HCC cells. Lithium administration can enhance pro-apoptotic chemotherapeutic drugs potential and overcome the resistance of tumor cells to apoptosis in HCC.

\section{ACKNOWLEDGMENT}

This work was supported with financing of the Novosibirsk Research Institute of Clinical and Experimental Lymphology as part of a state order, No. 0324-2019-0045.

\section{REFERENCES}

[1] W. K. Sung et al., "Genome-wide survey of recurrent HBV integration in hepatocellular carcinoma," Nat. Genet., vol. 44, pp. 765-769, 2012.

[2] I. Fabregat, "Dysregulation of apoptosis in hepatocellular carcinoma cells," World J. Gastroenterol., vol. 15, pp. 513-520, February 2009.

[3] J. Moreno-Càceres and I. Fabregat, "Apoptosis in liver carcinogenesis and chemotherapy," Hepat. Oncol., vol. 2, pp. 381-397, October 2015.

[4] I. S. Taskaeva and N. P. Bgatova, "Lithium salts in experimental oncology: review," Siberian Scientific Medical Journal, vol. 39, pp. 12-18, May 2019

[5] J. A. Quiroz, T. D. Gould and H. K. Manji, "Molecular effects of lithium," Mol. Interv., vol. 4, pp. 259-272, October 2004.

[6] Y. S. Gavrilova, N. P. Bgatova, A. O. Solov'eva, K. E. Trifonova, A. P. Lykov, Y. I. Borodin and V. I. Konenkov, "Target cells for lithium in different forms within a heterogeneous hepatocarcinoma-29 population," Cell Tiss. Biol., vol. 10, pp. 284-289, April 2016.

[7] N. P. Bgatova, Yu. S. Gavrilova, A. P. Lykov, A. O. Solovieva, V. V. Makarova, Yu. I. Borodin and V. I. Konenkov, "Apoptosis and autophagy in hepatocarcinoma cells induced by different forms of lithium salts," Cell Tiss. Biol., vol. 11, pp. 261-267, April 2017.

[8] V. I. Kaledin, N. A. Zhukova, V. P. Nikolin, N. A. Popova, M. D. Belyaev, N. V. Baginskaya, E. A. Litvinova, T. G. Tolstikova, E. L. Lushnikova and D. E. Semenov, "Hepatocellular carcinoma-29 metastatic transplantable tumor of mice, causing cachexia," Bull. Exp. Biol. Med., vol. 148, pp. 664-669, December 2009.

[9] L. Galluzzi, et al., "Molecular mechanisms of cell death: recommendations of the Nomenclature Committee on Cell Death 2018," Cell Death Differ., vol. 25, pp. 486-541, March 2018.

[10] L. Galluzzi, O. Kepp and G. Kroemer, "Mitochondrial regulation of cell death: a phylogenetically conserved control," Microb Cell, vol. 3, pp. 101-108, February 2016.

[11] E. Erdal, N. Ozturk, T. Cagatay, E. Eksioglu-Demiralp and M. Ozturk, "Lithium-mediated downregulation of PKB/Akt and cyclin E with growth inhibition in hepatocellular carcinoma cells," Int. J. Cancer, vol. 115, pp. 903-910, July 2005.

[12] E. Beurel, M. J. Blivet-Van Eggelpoël, M. Kornprobst, S. Moritz, R. Delelo, F. Paye, C. Housset and C. Desbois-Mouthon, "Glycogen synthase kinase-3 inhibitors augment TRAIL-induced apoptotic death in human hepatoma cells," Biochem. Pharmacol., vol. 77, pp. 54-65, January 2009 